ESAIM: COCV 19 (2013) 657-667

DOI: $10.1051 / \mathrm{cocv} / 2012026$
ESAIM: Control, Optimisation and Calculus of Variations

www.esaim-cocv.org

\title{
THE POLARIZATION IN A FERROELECTRIC THIN FILM: LOCAL AND NONLOCAL LIMIT PROBLEMS
}

\author{
Antonio Gaudiello $^{1}$ and Kamel Hamdache ${ }^{2}$
}

\begin{abstract}
In this paper, starting from classical non-convex and nonlocal $3 D$-variational model of the electric polarization in a ferroelectric material, via an asymptotic process we obtain a rigorous $2 D$-variational model for a thin film. Depending on the initial boundary conditions, the limit problem can be either nonlocal or local.
\end{abstract}

Mathematics Subject Classification. 35Q61, 78A25.

Received January 17, 2012. Revised May 22, 2012.

Published online March 28, 2013.

\section{INTRODUCTION}

Ferroelectric materials are polar materials that exhibit, at temperature below the Curie point, spontaneous electric polarization which can be reoriented using an applied electric field and a domain structure. In these materials, for temperature above the Curie point, the internal energy has a unique minimum point (paraelectric phase); whereas for the low temperature, it exhibits a double well profile and the polarization can be switched from a minimum point to the other (for instance, see [12]).

In this paper, for reasons of simplicity and economy, especially by a numerical point of view, we deal with $3 D-2 D$ dimensional reduction problems for ferroelectric materials.

Let

$$
\left.\Omega_{n}=\omega \times\right]-\frac{h_{n}}{2}, \frac{h_{n}}{2}[, \quad n \in \mathbb{N}
$$

be a $3 D$ ferroelectric device with open polygonal cross-section $\omega \subset \mathbb{R}^{2}$ and small thickness $h_{n}$, where $\left.h_{n} \in\right] 0,1[$, $n \in \mathbb{N}$, is a parameter tending to zero. In this material, the response to an applied electric field changes the electric displacement as $\mathbf{D}=\varepsilon E+4 \pi p$, where $\varepsilon>0$ is the dielectric permeability, $p$ is the spontaneous electric polarization field and $E=-D \varphi$ is the electric field which satisfies the electrostatic equation

$$
\begin{cases}\operatorname{div}(-\varepsilon D \varphi+4 \pi p)=0 & \text { in } \Omega_{n} \\ (-\varepsilon D \varphi+4 \pi p) \cdot \nu=0 & \text { on } \partial \Omega_{n}\end{cases}
$$

Keywords and phrases. Electric polarization, thin film, nonlocal problems.

1 DIEI, Università degli Studi di Cassino e del Lazio meridionale, via G. Di Biasio 43, 03043 Cassino (FR), Italia. gaudiell@unina.it

2 Centre de Mathématiques Appliquées, École Polytechnique, 91128 Palaiseau, France. kamel.hamdache@polytechnique.edu 
with $\nu$ denoting the unit outer normal on $\partial \Omega_{n}$. We remark that we do not discuss piezoelectric models since we are not considering deformations of the ferroelectric material. The free energy associated with $\Omega_{n}$ is non-convex, nonlocal and it is given by (for instance, see $[4,13,14]$ )

$$
\begin{aligned}
\mathcal{E}_{n}: p= & \left(p_{1}, p_{2}, p_{3}\right) \in\left(H^{1}\left(\Omega_{n}\right)\right)^{3} \rightarrow \frac{1}{h_{n}}\left[\beta \int_{\Omega_{n}}|\operatorname{rot} p|^{2} \mathrm{~d} x+\int_{\Omega_{n}}|\operatorname{div} p|^{2} \mathrm{~d} x+\right. \\
& \left.+\alpha \int_{\Omega_{n}}\left(|p|^{2}-1\right)^{2} \mathrm{~d} x+\int_{\Omega_{n}}|D \varphi|^{2} \mathrm{~d} x-\int_{\Omega_{n}}\left(g_{n} \cdot p\right) \mathrm{d} x\right]
\end{aligned}
$$

where $\alpha=-\frac{T-T_{c}}{T_{c}}$ is the reduced temperature which verifies $0<\alpha<1$ since temperature $T$ is assumed smaller than Curie temperature $T_{c}, \beta>0$ is a positive constant, $g_{n} \in\left(L^{2}\left(\Omega_{n}\right)\right)^{3}$ is an external electric field, $x=\left(x_{1}, x_{2}, x_{3}\right)=\left(x^{\prime}, x_{3}\right)$ denotes the generic point of $\mathbb{R}^{3}$ and $\cdot$ denotes the inner product in $\mathbb{R}^{3}$. We remark that the operator is anisotropic for $\beta \neq 1$.

After having imposed appropriate convergence assumptions on the rescaled exterior field in $\Omega=\omega \times]-\frac{1}{2}, \frac{1}{2}[$ (see (3.1) and (3.6)), the goal of this paper is to study the asymptotic behavior, as $h_{n}$ vanishes, of minimum of energy (1.2) with suitable boundary conditions, for obtaining a mathematical justification of the in-plane ferromagnetic model and of the uniaxial ferromagnetic model satisfied in a $2 D$ domain. To this aim, we consider two types of boundary conditions. Precisely, in the first case we assume

$$
p \cdot \nu=0 \quad \text { on } \quad \partial \Omega_{n}
$$

and we obtain (see Thm. 5.1) $\left(H^{1}(\Omega)\right)^{3}$-strong convergence for a subsequence of rescaled polarizations. The limit is independent of $x_{3}$, parallel to cross-section $\omega$ and the in-plane components solve in $\omega$ a problem analogous to the original problem with an external field given by the average, in $x_{3}$-direction, of the first two components of the $\left(L^{2}(\Omega)\right)^{3}$-weak limit of the rescaled external field. Moreover, we obtain also $\left(H^{1}(\Omega)\right)$-strong convergence for the rescaled potential of electric field $E$ associated with the polarization and the convergence of the energies. In the second case, we assume

$$
p \wedge \nu=0 \quad \text { on } \quad \partial \Omega_{n},
$$

with $\wedge$ denoting the cross product in $\mathbb{R}^{3}$. Also in this case we obtain (see Thm. 5.3) $\left(H^{1}(\Omega)\right)^{3}$-strong convergence for a subsequence of rescaled polarizations with a limit independent of $x_{3}$, but now it is orthogonal to $\omega$ and the out-plane component solves in $\omega$ the following local problem

$$
\min \left\{\int_{\omega}\left[\beta|D q|^{2}+\alpha\left(|q|^{2}-1\right)^{2}+\left(\frac{4 \pi}{\varepsilon}\right)^{2}|q|^{2}-g_{3} q\right] \mathrm{d} x^{\prime}: q \in H_{0}^{1}(\omega)\right\},
$$

where $g_{3}$ is the average, in $x_{3}$-direction, of the third component of the $\left(L^{2}(\Omega)\right)^{3}$-weak limit of the rescaled external field. Moreover, we prove that the rescaled potential of electric field $E$ associated with the polarization converges to zero $\left(H^{1}(\Omega)\right)$-strongly and we prove also the convergence of the energies. In the sequel, for simplifying computations, we assume $\varepsilon=4 \pi$.

We remark that both boundary conditions (1.3) and (1.4) allow to estimate $\|D p\|_{\left(L^{2}\left(\Omega_{n}\right)\right)^{9}}$ in terms of $\|\operatorname{rot} p\|_{\left(L^{2}\left(\Omega_{n}\right)\right)^{3}}$ and $\|\operatorname{div} p\|_{L^{2}\left(\Omega_{n}\right)}$, independently of $n \in N$, and to obtain a priori estimates on the polarization. Just for simplifying the proof of this point, we assume that $\Omega_{n}$ is a polygon. We remark that a priori estimates are not evident without assumption (1.3) or (1.4). We reformulate our problem on a fixed domain through appropriate rescalings of the kind proposed by Ciarlet and Destuynder [5] and impose appropriate convergence assumptions on the rescaled exterior fields. According to the boundary conditions, the limit of the rescaled polarization is parallel or orthogonal to $\omega$. This information plays an essential role in describing the limit behavior of the rescaled potential of electric field $E$ (see Prop. (4.1)). We conclude using the main idea of $\Gamma$-convergence method introduced by De Giorgi and Franzoni [7]. 
Our analysis is close to that of ferromagnetic thin films. In what concerns the study of a ferromagnetic thin film, several results are present in literature. Gioia and James [10] were the first to prove that the magnetostatic energy behaves, at the limit, like an anisotropic local term which forces the magnetization to be tangent to the thin film. The time-dependent case was treated in [2] and in [3]. In [1] the authors proposed a model of films with strong convergence of minimizers when the exchange parameter vanishes and with vertically invariant configurations on the cylindrical domain. For reproducing the non uniform states observed experimentally in thin films, very different regimes were considered in [8] and in [11], where $\frac{h}{l}$ and $\frac{\lambda}{l}$ vanish, $h$ being the film thickness, $l$ the in-plane diameter and $\lambda$ the exchange length of the ferromagnetic material. Joined ferromagnetic thin films were recently studied in [9].

\section{The SETting of THE PROBLEMS}

For every $n \in \mathbb{N}$, let

$$
P_{n}=\left\{p \in\left(H^{1}\left(\Omega_{n}\right)\right)^{3}: p \cdot \nu=0 \text { on } \partial \Omega_{n}\right\} \text { and } S_{n}=\left\{p \in\left(H^{1}\left(\Omega_{n}\right)\right)^{3}: p \wedge \nu=0 \text { on } \partial \Omega_{n}\right\} .
$$

Lemma 2.1. It results that

$$
\|D p\|_{\left(L^{2}\left(\Omega_{n}\right)\right)^{9}}^{2}=\|\operatorname{rot} p\|_{\left(L^{2}\left(\Omega_{n}\right)\right)^{3}}^{2}+\|\operatorname{div} p\|_{L^{2}\left(\Omega_{n}\right)}^{2}, \forall p \in P_{n} \cup S_{n}, \forall n \in \mathbb{N} .
$$

Proof. It is known that this equality holds true in $\left(P_{n} \cup S_{n}\right) \cap\left(H^{\infty}\left(\Omega_{n}\right)\right)^{3}$ (see the Proof of Lem. 2.2 in [6]). Consequently, using the density of $P_{n} \cap\left(H^{\infty}\left(\Omega_{n}\right)\right)^{3}$ in $P_{n}$ and the density of $S_{n} \cap\left(H^{\infty}\left(\Omega_{n}\right)\right)^{3}$ in $S_{n}$ (see Lem. 2.6 in $[6])$, one obtains $(2.1)$.

For every $n \in \mathbb{N}$ and $p \in\left(L^{2}\left(\Omega_{n}\right)\right)^{3}$, Lax-Milgram theorem ensures that the following problem

$$
\varphi_{p} \in H^{1}\left(\Omega_{n}\right), \quad \int_{\Omega_{n}} \varphi_{p} \mathrm{~d} x=0, \quad \int_{\Omega_{n}}\left(\left(-D \varphi_{p}+p\right) \cdot D \varphi\right) \mathrm{d} x=0, \quad \forall \varphi \in H^{1}\left(\Omega_{n}\right),
$$

admits a unique solution.

For every $n \in N$, let

$$
\mathcal{E}_{n}: p \in\left(H^{1}\left(\Omega_{n}\right)\right)^{3} \rightarrow \frac{1}{h_{n}} \int_{\Omega_{n}}\left[\beta|\operatorname{rot} p|^{2}+|\operatorname{div} p|^{2}+\alpha\left(|p|^{2}-1\right)^{2}+\left|D \varphi_{p}\right|^{2}-\left(g_{n} \cdot p\right)\right] \mathrm{d} x,
$$

where $g_{n} \in\left(L^{2}\left(\Omega_{n}\right)\right)^{3}$ and $\varphi_{p}$ is the unique solution of (2.2). By using (2.1) and the direct method of Calculus of Variations, it is easy to see that the following problems

$$
\begin{aligned}
& \min \left\{\mathcal{E}_{n}(p): p \in P_{n}\right\}, \\
& \min \left\{\mathcal{E}_{n}(p): p \in S_{n}\right\}
\end{aligned}
$$

admit solution. The aim of this paper is to study the asymptotic behavior, as $n$ diverges, of problems (2.4) and (2.5). The natural space for studying problem (2.4) and (2.5) would be

$$
\Pi_{n}=\left\{p \in H\left(\text { div, curl, } \Omega_{n}\right): p \cdot \nu=0 \text { on } \partial \Omega_{n}\right\} \text { and } \Sigma_{n}=\left\{p \in H\left(\operatorname{div}, \operatorname{curl}, \Omega_{n}\right): p \wedge \nu=0 \text { on } \partial \Omega_{n}\right\},
$$

respectively. On the other side, Lemma 2.1 provides that $\Pi_{n}=P_{n}$ and $\Sigma_{n}=S_{n}$. 


\section{The RESCALing}

As it is usual (see [5]), problems (2.4) and (2.5) can be reformulated on a fixed domain through the following rescaling:

$$
\left.x=\left(x_{1}, x_{2}, x_{3}\right) \in \Omega=\omega \times\right]-\frac{1}{2}, \frac{1}{2}\left[\rightarrow\left(x_{1}, x_{2}, h_{n} x_{3}\right) \in \Omega_{n}=\omega \times\right]-\frac{h_{n}}{2}, \frac{h_{n}}{2}[.
$$

Precisely, setting

$$
P=\left\{p \in\left(H^{1}(\Omega)\right)^{3}: p \cdot \nu=0 \text { on } \partial \Omega\right\} \text { and } S=\left\{p \in\left(H^{1}(\Omega)\right)^{3}: p \wedge \nu=0 \text { on } \partial \Omega\right\}
$$

where $\nu$ denotes also the unit outer normal on $\partial \Omega$, and for every $n \in N$

$$
\begin{gathered}
f_{n}: x=\left(x_{1}, x_{2}, x_{3}\right) \in \Omega \rightarrow g_{n}\left(x_{1}, x_{2}, h_{n} x_{3}\right), \\
D_{n}: p \in\left(H^{1}(\Omega)\right)^{3}\left(\operatorname{resp.} H^{1}(\Omega)\right) \rightarrow\left(\frac{\partial p}{\partial x_{1}}, \frac{\partial p}{\partial x_{2}}, \frac{1}{h_{n}} \frac{\partial p}{\partial x_{3}}\right) \in\left(L^{2}(\Omega)\right)^{9} \quad\left(\text { resp. }\left(L^{2}(\Omega)\right)^{3}\right), \\
\operatorname{div}_{n}: p=\left(p_{1}, p_{2}, p_{3}\right) \in\left(H^{1}(\Omega)\right)^{3} \rightarrow \frac{\partial p_{1}}{\partial x_{1}}+\frac{\partial p_{2}}{\partial x_{2}}+\frac{1}{h_{n}} \frac{\partial p_{3}}{\partial x_{3}} \in L^{2}(\Omega), \\
\operatorname{rot}_{n}: p=\left(p_{1}, p_{2}, p_{3}\right) \in\left(H^{1}(\Omega)\right)^{3} \rightarrow\left(\frac{\partial p_{3}}{\partial x_{2}}-\frac{1}{h_{n}} \frac{\partial p_{2}}{\partial x_{3}}, \frac{1}{h_{n}} \frac{\partial p_{1}}{\partial x_{3}}-\frac{\partial p_{3}}{\partial x_{1}}, \frac{\partial p_{2}}{\partial x_{1}}-\frac{\partial p_{1}}{\partial x_{2}}\right) \in\left(L^{2}(\Omega)\right)^{3},
\end{gathered}
$$

functional $\mathcal{E}_{n}$ defined in (2.3) is rescaled in the following one:

$$
E_{n}: p \in\left(H^{1}(\Omega)\right)^{3} \rightarrow \int_{\Omega}\left[\beta\left|\operatorname{rot}_{n} p\right|^{2}+\left|\operatorname{div}_{n} p\right|^{2}+\alpha\left(|p|^{2}-1\right)^{2}+\left|D_{n} \phi_{p}\right|^{2}-\left(f_{n} \cdot p\right)\right] \mathrm{d} x,
$$

where $\phi_{p}$ is the unique solution of the following problem

$$
\phi_{p} \in H^{1}(\Omega), \quad \int_{\Omega} \phi_{p} \mathrm{~d} x=0, \quad \int_{\Omega}\left(\left(-D_{n} \phi_{p}+p\right) \cdot D_{n} \phi\right) \mathrm{d} x=0, \quad \forall \phi \in H^{1}(\Omega),
$$

which rescales problem (2.2). Then, the goal of this paper turns in studying the asymptotic behavior, as $n$ diverges, of the following problems

$$
\begin{aligned}
& \min \left\{E_{n}(p): p \in P\right\}, \\
& \min \left\{E_{n}(p): p \in S\right\} .
\end{aligned}
$$

To this aim, we assume that

$$
f_{n} \rightarrow f=\left(f_{1}, f_{2}, f_{3}\right) \text { weakly in }\left(L^{2}(\Omega)\right)^{3} .
$$

We conclude this section recalling that (2.1) transforms into the following one:

$$
\left\|D_{n} p\right\|_{\left(L^{2}(\Omega)\right)^{9}}^{2}=\left\|\operatorname{rot}_{n} p\right\|_{\left(L^{2}(\Omega)\right)^{3}}^{2}+\left\|\operatorname{div}_{n} p\right\|_{L^{2}(\Omega)}^{2}, \forall p \in P \cup S, \forall n \in \mathbb{N} .
$$

\section{A CONVERGENCE RESUlt FOR PROBlem (3.3)}

Proposition 4.1. Let $\left\{p_{n}\right\}_{n \in \mathbb{N}} \subset\left(L^{2}(\Omega)\right)^{3}$ be such that

$$
p_{n} \rightarrow q \text { strongly in }\left(L^{2}(\Omega)\right)^{3},
$$

with $q=\left(q_{1}, q_{2}, q_{3}\right)=\left(q^{\prime}, q_{3}\right) \in\left(L^{2}(\Omega)\right)^{3}$ independent of $x_{3}$. Moreover, let $\phi_{p_{n}}$ be the unique solution of (3.3) 
with $p=p_{n}$. Then,

(i) if $q_{3}=0$, it results that

$$
\begin{gathered}
\phi_{p_{n}} \rightarrow \psi_{q^{\prime}} \text { strongly in } H^{1}(\Omega), \\
\frac{1}{h_{n}} \frac{\partial \phi_{p_{n}}}{\partial x_{3}} \rightarrow 0 \text { strongly in } L^{2}(\Omega),
\end{gathered}
$$

where $\psi_{q^{\prime}}$ is the unique solution of the following problem

$$
\psi_{q^{\prime}} \in H^{1}(\omega), \quad \int_{\omega} \psi_{q^{\prime}} \mathrm{d} x^{\prime}=0, \quad \int_{\omega}\left(\left(-D \psi_{q^{\prime}}+q^{\prime}\right) \cdot D \psi\right) \mathrm{d} x^{\prime}=0, \quad \forall \psi \in H^{1}(\omega) ;
$$

(ii) if $q^{\prime}=0$, it results that

$$
\begin{gathered}
\phi_{p_{n}} \rightarrow 0 \text { strongly in } H^{1}(\Omega), \\
\frac{1}{h_{n}} \frac{\partial \phi_{p_{n}}}{\partial x_{3}} \rightarrow q_{3} \text { strongly in } L^{2}(\Omega) .
\end{gathered}
$$

Proof. Since $\left\{p_{n}\right\}_{n \in \mathbb{N}}$ is bounded in $\left(L^{2}(\Omega)\right)^{3}$, there exists a positive constant $c$ such that

$$
\left\|D_{n} \phi_{p_{n}}\right\|_{\left(L^{2}(\Omega)\right)^{3}} \leq c, \quad \forall n \in \mathbb{N} .
$$

Consequently, by virtue of the Poincaré-Wirtinger inequality, there exist a subsequence of $\mathbb{N}$, still denoted by $\{n\}$, and (in possible dependence of the subsequence) $\tau \in H^{1}(\Omega)$, independent of $x_{3}$ and with zero average, and $\xi \in L^{2}(\Omega)$ such that

$$
\begin{gathered}
\phi_{p_{n}} \rightarrow \tau \text { weakly in } H^{1}(\Omega), \\
\frac{1}{h_{n}} \frac{\partial \phi_{p_{n}}}{\partial x_{3}} \rightarrow \xi \text { weakly in } L^{2}(\Omega) .
\end{gathered}
$$

Now, we prove the first case, that is we assume $q_{3}=0$. By passing to the limit in the equation satisfied by $\phi_{p_{n}}$ with test functions $\psi$ independent of $x_{3}$, that is $\psi \in H^{1}(\omega)$, and recalling that $q^{\prime}$ is independent of $x_{3}$, convergences (4.1) and (4.7) entail that

$$
\tau \in H^{1}(\omega), \quad \int_{\omega} \tau \mathrm{d} x^{\prime}=0, \quad \int_{\omega}\left(\left(-D \tau+q^{\prime}\right) \cdot D \psi\right) \mathrm{d} x^{\prime}=0, \quad \forall \psi \in H^{1}(\omega)
$$

Since this equation admits a unique solution $\psi_{q^{\prime}}$, it results that $\tau=\psi_{q^{\prime}}$, that is

$$
\phi_{p_{n}} \rightarrow \psi_{q^{\prime}} \text { weakly in } H^{1}(\Omega) \text {. }
$$

By using (4.10), (4.8), a l.s.c. argument, (4.1) and (4.9), one obtains that

$$
\int_{\omega}\left|D \psi_{q^{\prime}}\right|^{2} \mathrm{~d} x^{\prime}+\int_{\Omega}|\xi|^{2} \mathrm{~d} x \leq \lim _{n} \int_{\Omega}\left|D_{n} \phi_{p_{n}}\right|^{2} \mathrm{~d} x=\lim _{n} \int_{\Omega}\left(D_{n} \phi_{p_{n}} \cdot p_{n}\right) \mathrm{d} x=\int_{\omega}\left(D \psi_{q^{\prime}} \cdot q^{\prime}\right) \mathrm{d} x^{\prime}=\int_{\omega}\left|D \psi_{q^{\prime}}\right|^{2} \mathrm{~d} x^{\prime},
$$

which provides that $\xi=0$ and that convergences (4.8) and (4.10) are strong. We notice that convergences (4.2) and (4.3) hold true for the whole sequence since the limits are uniquely identified.

Now, we prove the second case, that is we assume $q^{\prime}=0$. By passing to the limit in the equation satisfied by $\phi_{p_{n}}$ with test functions $\psi$ independent of $x_{3}$, that is $\psi \in H^{1}(\omega)$, convergences (4.1) and (4.7) entail that

$$
\tau \in H^{1}(\omega), \quad \int_{\omega} \tau \mathrm{d} x^{\prime}=0, \quad \int_{\omega}(D \tau \cdot D \psi) \mathrm{d} x^{\prime}=0, \quad \forall \psi \in H^{1}(\omega)
$$

that is $\tau=0$. 
Now, passing to the limit in the equation satisfied by $\phi_{p_{n}}$ with test functions $h_{n} \phi, \phi \in H^{1}(\Omega)$, by virtue of (4.1), (4.7) and (4.8) one obtains that

$$
\int_{\Omega}\left(-\xi+q_{3}\right) \frac{\partial \phi}{\partial x_{3}}=0, \quad \forall \phi \in H^{1}(\Omega)
$$

from which, taking into account that $q_{3}$ is independent of $x_{3}$, it follows that also $\xi$ is independent of $x_{3}$. Consequently, choosing in (4.12) $\phi=x_{3} \psi$ with $\psi \in C_{0}^{\infty}(\omega)$, it results that $\xi=q_{3}$. One concludes the proof arguing as in the first case.

\section{The MAIN RESULtS}

At first, we consider the case $p \cdot \nu=0$ on $\partial \Omega$. Let

$$
\begin{aligned}
P_{\infty} & =\left\{q \in\left(H^{1}(\Omega)\right)^{2}: q \text { is independent of } x_{3} \text { and } q \cdot \nu^{\prime}=0 \text { on } \partial \omega \times\right]-\frac{1}{2}, \frac{1}{2}[\} \\
& \simeq\left\{q \in\left(H^{1}(\omega)\right)^{2}: q \cdot \nu^{\prime}=0 \text { on } \partial \omega\right\}
\end{aligned}
$$

and

$E_{\infty}: q \in P_{\infty} \rightarrow \beta \int_{\omega}|\operatorname{rot} q|^{2} \mathrm{~d} x^{\prime}+\int_{\omega}|\operatorname{div} q|^{2} \mathrm{~d} x^{\prime}+\alpha \int_{\omega}\left(|q|^{2}-1\right)^{2} \mathrm{~d} x^{\prime}+\int_{\omega}\left|D \psi_{q}\right|^{2} \mathrm{~d} x^{\prime}-\int_{\omega}\left(\int_{-\frac{1}{2}}^{-\frac{1}{2}}\left(f_{1}, f_{2}\right) \mathrm{d} x_{3} \cdot q\right) \mathrm{d} x^{\prime}$,

where $\left(f_{1}, f_{2}\right)$ is defined in (3.6), $\psi_{q}$ is the unique solution of (4.4) and, for $q=\left(q_{1}, q_{2}\right), \operatorname{rot}\left(q_{1}, q_{2}\right)=\frac{\partial q_{2}}{\partial x_{1}}-\frac{\partial q_{1}}{\partial x_{2}}$.

Theorem 5.1. Assume (3.6). For every $n \in \mathbb{N}$, let $E_{n}$ be defined in (3.2), $p_{n}$ be a solution of (3.4) and $\phi_{p_{n}}$ be the unique solution of (3.3) with $p=p_{n}$. Moreover, let $P_{\infty}$ and $E_{\infty}$ be defined in (5.1) and (5.2), respectively. Then, there exist an increasing sequence of positive integer numbers $\left\{n_{i}\right\}_{i \in \mathbb{N}}$ and (in possible dependence of the subsequence) $\bar{q} \in P_{\infty}$ such that

$$
\begin{gathered}
p_{n_{i}} \rightarrow(\bar{q}, 0) \text { strongly in }\left(H^{1}(\Omega)\right)^{3} \text { and strongly in }\left(L^{4}(\Omega)\right)^{3}, \\
\frac{1}{h_{n}} \frac{\partial p_{n}}{\partial x_{3}} \rightarrow 0 \text { strongly in }\left(L^{2}(\Omega)\right)^{3} \\
\phi_{p_{n_{i}}} \rightarrow \psi_{\bar{q}} \text { strongly in } H^{1}(\Omega) \\
\frac{1}{h_{n}} \frac{\partial \phi_{p_{n}}}{\partial x_{3}} \rightarrow 0 \text { strongly in } L^{2}(\Omega)
\end{gathered}
$$

where $\bar{q}$ is a solution of the following problem

$$
E_{\infty}(\bar{q})=\min \left\{E_{\infty}(q): q \in P_{\infty}\right\}
$$

and $\psi_{\bar{q}}$ is the unique solution of (4.4) with $q^{\prime}=\bar{q}$. Moreover, the convergence of the energies holds true, that is

$$
\lim _{n} E_{n}\left(p_{n}\right)=E_{\infty}(\bar{q})
$$


Proof. The proof of this theorem will be performed in several steps. The first step is devoted to prove that there exist a subsequence of $\mathbb{N}$, still denoted by $\{n\}$, and (in possible dependence of the subsequence) $\bar{q} \in P_{\infty}$ and $z \in\left(L^{2}\left(\omega, H_{m}^{1}(]-\frac{1}{2}, \frac{1}{2}[)\right)\right)^{2} \times L^{2}\left(\omega, H_{0}^{1}(]-\frac{1}{2}, \frac{1}{2}[)\right)$ such that

$$
\begin{gathered}
p_{n} \rightarrow(\bar{q}, 0) \text { weakly in }\left(H^{1}(\Omega)\right)^{3} \text { and strongly in }\left(L^{4}(\Omega)\right)^{3}, \\
\frac{1}{h_{n}} \frac{\partial p_{n}}{\partial x_{3}} \rightarrow \frac{\partial z}{\partial x_{3}} \text { weakly in }\left(L^{2}(\Omega)\right)^{3},
\end{gathered}
$$

where the subscript " $m$ " means zero average.

Since $0 \in P$, it results that

$$
\int_{\Omega}\left(\beta\left|\operatorname{rot}_{n} p_{n}\right|^{2}+\left|\operatorname{div}_{n} p_{n}\right|^{2}+\alpha\left|p_{n}\right|^{4}-2 \alpha\left|p_{n}\right|^{2}\right) \mathrm{d} x \leq\left\|f_{n}\right\|_{\left(L^{2}(\Omega)\right)^{3}}\left\|p_{n}\right\|_{\left(L^{2}(\Omega)\right)^{3}}, \forall n \in \mathbb{N} .
$$

By using the continuous embedding of $\left(L^{4}(\Omega)\right)^{3}$ into $\left(L^{2}(\Omega)\right)^{3}$, estimate $(5.11)$ gives

$$
\frac{\alpha}{|\Omega|}\left\|p_{n}\right\|_{L^{2}(\Omega)}^{3}-2 \alpha\left\|p_{n}\right\|_{L^{2}(\Omega)} \leq\left\|f_{n}\right\|_{\left(L^{2}(\Omega)\right)^{3}}, \quad \forall n \in \mathbb{N},
$$

from which, by virtue of (3.6), it follows the existence of a positive constant $c$ such that

$$
\left\|p_{n}\right\|_{\left(L^{2}(\Omega)\right)^{3}} \leq c, \quad \forall n \in \mathbb{N} .
$$

Then, combining (5.13), (3.6), (5.11) and (3.7), one obtains also the existence of a positive constant $c$ such that

$$
\left\|D_{n} p_{n}\right\|_{\left(L^{2}(\Omega)\right)^{9}} \leq c, \quad \forall n \in \mathbb{N} .
$$

Estimates (5.13) and (5.14) provide the existence of a subsequence of $\mathbb{N}$, still denoted by $\{n\}$, and (in possible dependence of the subsequence) $\bar{p}=\left(\bar{p}_{1}, \bar{p}_{2}, \bar{p}_{3}\right) \in P$, independent of $x_{3}$, such that

$$
p_{n} \rightarrow \bar{p} \text { weakly in }\left(H^{1}(\Omega)\right)^{3} \text { and strongly in }\left(L^{4}(\Omega)\right)^{3} .
$$

In particular, since $\bar{p}_{3}$ is independent of $x_{3}$ and $\bar{p} \cdot \nu=0$ on $\partial \Omega$, it results that $\bar{p}_{3}=0$. Then, setting $\left(\bar{p}_{1}, \bar{p}_{2}\right)=\bar{q}$, one has that $\bar{q} \in P_{\infty}$ and (5.9) holds true.

To prove (5.10), for $i=1,2$ and for every $n \in \mathbb{N}$ set

$$
m_{n, i}: x^{\prime} \in \omega \longrightarrow \int_{-\frac{1}{2}}^{\frac{1}{2}} p_{n, i}\left(x^{\prime}, x_{3}\right) \mathrm{d} x_{3} .
$$

By using the Poincaré-Wirtinger inequality and recalling that $p_{n} \cdot \nu=0$ on $\partial \Omega$ entails $p_{n, 3}\left(x_{1}, x_{2}, \cdot\right) \in$ $H_{0}^{1}(]-\frac{1}{2}, \frac{1}{2}[)$, one obtains the existence of a positive constant $c$ such that, for $x^{\prime}$ a.e. in $\omega$,

$$
\begin{gathered}
\left\|\frac{1}{h_{n}}\left(p_{n, i}\left(x^{\prime}, \cdot\right)-m_{n, i}\left(x^{\prime}\right)\right)\right\|_{H_{m}^{1}(]-\frac{1}{2}, \frac{1}{2}[)} \leq \frac{c}{h_{n}}\left\|\frac{\partial p_{n, i}\left(x^{\prime}, \cdot\right)}{\partial x_{3}}\right\|_{L^{2}(]-\frac{1}{2}, \frac{1}{2}[)}, \quad \forall n \in \mathbb{N}, \quad i=1,2, \\
\left\|\frac{1}{h_{n}} p_{n, 3}\left(x^{\prime}, \cdot\right)\right\|_{H_{0}^{1}(]-\frac{1}{2}, \frac{1}{2}[)} \leq \frac{c}{h_{n}}\left\|\frac{\partial p_{n, 3}\left(x^{\prime}, \cdot\right)}{\partial x_{3}}\right\|_{L^{2}(]-\frac{1}{2}, \frac{1}{2}[)}, \quad \forall n \in \mathbb{N} .
\end{gathered}
$$

Thus, integrating these inequalities over $x^{\prime} \in \omega$, estimate (5.14) gives (5.10).

The second step is devoted to identify $\bar{q}$ and $z$. Since $\left(q_{1}, q_{2}, 0\right) \in P$ for every $q=\left(q_{1}, q_{2}\right) \in P_{\infty}$, it results that

$$
E_{n}\left(p_{n}\right) \leq E_{n}\left(\left(q_{1}, q_{2}, 0\right)\right), \quad \forall q=\left(q_{1}, q_{2}\right) \in P_{\infty}, \quad \forall n \in \mathbb{N} .
$$


Then, passing to the limit in (5.15), by virtue of (3.6), Proposition 4.1, (5.9), (5.10) and a l.s.c. argument one obtains that

$$
\begin{aligned}
\beta \int_{\Omega}\left(\left|\frac{\partial z_{2}}{\partial x_{3}}\right|^{2}+\left|\frac{\partial z_{1}}{\partial x_{3}}\right|^{2}\right) \mathrm{d} x+\beta \int_{\omega}|\operatorname{rot} \bar{q}|^{2} \mathrm{~d} x^{\prime}+\int_{\Omega}\left|\operatorname{div} \bar{q}+\frac{\partial z_{3}}{\partial x_{3}}\right|^{2} \mathrm{~d} x \\
+\alpha \int_{\omega}\left(|\bar{q}|^{2}-1\right)^{2} \mathrm{~d} x^{\prime}+\int_{\omega}\left|D \psi_{\bar{q}}\right|^{2} \mathrm{~d} x^{\prime}-\int_{\omega}\left(\int_{-\frac{1}{2}}^{\frac{1}{2}}\left(f_{1}, f_{2}\right) \mathrm{d} x_{3} \cdot \bar{q}\right) \mathrm{d} x^{\prime} \\
\quad \leq \underset{n}{ } \liminf _{n} E_{n}\left(p_{n}\right) \leq \limsup _{n} E_{n}\left(p_{n}\right) \leq E_{\infty}(q), \quad \forall q \in P_{\infty} .
\end{aligned}
$$

On the other hand, since $\bar{q}$ is independent of $x_{3}$ and $z_{3} \in L^{2}\left(\omega, H_{0}^{1}(]-\frac{1}{2}, \frac{1}{2}[)\right)$, it results that

$$
\int_{\Omega}\left|\operatorname{div} \bar{q}+\frac{\partial z_{3}}{\partial x_{3}}\right|^{2} \mathrm{~d} x=\int_{\omega}|\operatorname{div} \bar{q}|^{2} \mathrm{~d} x^{\prime}+\int_{\Omega}\left|\frac{\partial z_{3}}{\partial x_{3}}\right|^{2} \mathrm{~d} x .
$$

Hence, inserting (5.17) in (5.16), one has that

$$
c \int_{\Omega}\left|\frac{\partial z}{\partial x_{3}}\right|^{2} \mathrm{~d} x+E_{\infty}(\bar{q}) \leq \liminf _{n} E_{n}\left(p_{n}\right) \leq \limsup _{n} E_{n}\left(p_{n}\right) \leq E_{\infty}(q), \quad \forall q \in P_{\infty},
$$

where $c=\min \{1, \beta\}$, which entails that

$$
\frac{\partial z}{\partial x_{3}}=0, \text { a.e. in } \Omega
$$

(in particular, $z=0$ a.e. in $\Omega$ since $\left.z \in\left(L^{2}\left(\omega, H_{m}^{1}(]-\frac{1}{2}, \frac{1}{2}[)\right)\right)^{2} \times L^{2}\left(\omega, H_{0}^{1}(]-\frac{1}{2}, \frac{1}{2}[)\right)\right)$. Consequently, inserting (5.19) in (5.18), one obtains that $\bar{q}$ solves problem (5.7) and convergence (5.8) holds true. We notice that convergences in (5.8) and (5.10) hold true for the whole sequence since the limits are uniquely identified. Moreover, (5.5) and (5.6) follow from (5.9) and Proposition 4.1.

It remains to prove that convergences in (5.9) and (5.10) are strong. To this aim, by combining (5.8) with (3.6), (5.5), (5.6) and (5.9), one obtains that

$$
\lim _{n} \int_{\Omega}\left(\beta\left|\operatorname{rot}_{n} p_{n}\right|^{2}+\left|\operatorname{div}_{n} p_{n}\right|^{2}\right) \mathrm{d} x=\int_{\Omega}\left(\beta|\operatorname{rot}(\bar{q}, 0)|^{2}+|\operatorname{div}(\bar{q}, 0)|^{2}\right) \mathrm{d} x,
$$

from which, using (5.9), (5.10) and (5.19), it follows that

$$
\operatorname{rot}_{n} p_{n} \rightarrow \operatorname{rot}(\bar{q}, 0) \text { strongly in }\left(L^{2}(\Omega)\right)^{3}, \quad \operatorname{div}_{n} p_{n} \rightarrow \operatorname{div}(\bar{q}, 0) \text { strongly in } L^{2}(\Omega) .
$$

Finally, combining (5.20) with (3.7), one derives that

$$
D_{n} p_{n} \rightarrow D(\bar{q}, 0) \text { strongly in }\left(L^{2}(\Omega)\right)^{9},
$$

which provides that convergences in (5.9) and (5.10) are strong.

Remark 5.2. Let $\bar{P}_{\infty}=P_{\infty} \times\{0\}$ and $\bar{E}_{\infty}(p)=\left\{\begin{array}{l}E_{\infty}(p) \text { in } \bar{P}_{\infty}, \\ +\infty \text { in } P \backslash \bar{P}_{\infty} .\end{array}\right.$ Then, $\Gamma$-convergence of $E_{\left.n\right|_{P}}$ to $\bar{E}_{\infty}$ in the strong topology of $\left(L^{2}(\Omega)\right)^{3}$ follows easily from previous proofs.

Now, we consider the case $p \wedge \nu=0$ on $\partial \Omega$. Let

$$
M_{\infty}: q \in H_{0}^{1}(\omega) \rightarrow \int_{\omega}\left(\beta|D q|^{2}+\alpha\left(|q|^{2}-1\right)^{2}+|q|^{2}-\left(\int_{-\frac{1}{2}}^{-\frac{1}{2}} f_{3} \mathrm{~d} x_{3} q\right)\right) \mathrm{d} x^{\prime},
$$

where $f_{3}$ is defined in (3.6). 
Theorem 5.3. Assume (3.6). For every $n \in \mathbb{N}$, let $E_{n}$ be defined in (3.2), $p_{n}$ be a solution of (3.5) and $\phi_{p_{n}}$ be the unique solution of (3.3) with $p=p_{n}$. Moreover, let $M_{\infty}$ be defined in (5.21). Then, there exist an increasing sequence of positive integer numbers $\left\{n_{i}\right\}_{i \in \mathbb{N}}$ and (in possible dependence of the subsequence) $\bar{q} \in\left\{q \in H^{1}(\Omega): q\right.$ independent of $x_{3}, q=0$ on $\left.\partial \omega \times\right]-\frac{1}{2}, \frac{1}{2}[\} \simeq H_{0}^{1}(\omega)$ such that

$$
\begin{gathered}
p_{n_{i}} \rightarrow(0,0, \bar{q}) \text { strongly in }\left(H^{1}(\Omega)\right)^{3} \text { and strongly in }\left(L^{4}(\Omega)\right)^{3}, \\
\qquad \begin{array}{c}
\frac{1}{h_{n}} \frac{\partial p_{n}}{\partial x_{3}} \rightarrow 0 \text { strongly in }\left(L^{2}(\Omega)\right)^{3}, \\
\phi_{p_{n}} \rightarrow 0 \text { strongly in } H^{1}(\Omega), \\
\frac{1}{h_{n_{i}}} \frac{\partial \phi_{p_{n_{i}}}}{\partial x_{3}} \rightarrow \bar{q} \text { strongly in } L^{2}(\Omega),
\end{array}
\end{gathered}
$$

where $\bar{q}$ is a solution of the following problem:

$$
M_{\infty}(\bar{q})=\min \left\{M_{\infty}(q): q \in H_{0}^{1}(\omega)\right\} .
$$

Moreover, the convergence of the energies hold true, that is

$$
\lim _{n} E_{n}\left(p_{n}\right)=M_{\infty}(\bar{q})
$$

Proof. We sketch the proof. The first step is devoted to prove that there exist a subsequence of $\mathbb{N}$, still denoted by $\{n\}$, and (in possible dependence of the subsequence) $\bar{q} \in$ $\left\{q \in H^{1}(\Omega): q\right.$ independent of $x_{3}, q=0$ on $\left.\partial \omega \times\right]-\frac{1}{2}, \frac{1}{2}[\}$ and $z \in\left(L^{2}\left(\omega, H_{0}^{1}(]-\frac{1}{2}, \frac{1}{2}[)\right)\right)^{2} \times L^{2}\left(\omega, H_{m}^{1}(]-\right.$ $\left.\frac{1}{2}, \frac{1}{2}[)\right)$ such that

$$
\begin{gathered}
p_{n} \rightarrow(0,0, \bar{q}) \text { weakly in }\left(H^{1}(\Omega)\right)^{3} \text { and strongly in }\left(L^{4}(\Omega)\right)^{3}, \\
\frac{1}{h_{n}} \frac{\partial p_{n}}{\partial x_{3}} \rightarrow \frac{\partial z}{\partial x_{3}} \text { weakly in }\left(L^{2}(\Omega)\right)^{3} .
\end{gathered}
$$

By using (3.7) and arguing as in the beginning of the Proof of Proposition 5.1, one can prove the existence a positive constant $c$ such that

$$
\left\|D_{n} p_{n}\right\|_{\left(L^{2}(\Omega)\right)^{9}} \leq c, \quad \forall n \in \mathbb{N},
$$

and the existence of a subsequence of $\mathbb{N}$, still denoted by $\{n\}$, and (in dependence of the possible subsequence) $\bar{p}=\left(\bar{p}_{1}, \bar{p}_{2}, \bar{p}_{3}\right) \in S$, independent of $x_{3}$, such that

$$
p_{n} \rightarrow \bar{p} \text { weakly in }\left(H^{1}(\Omega)\right)^{3} \text { and strongly in }\left(L^{4}(\Omega)\right)^{3} .
$$

In particular, since $\bar{p}$ is independent of $x_{3}$ and $\bar{p} \wedge \nu=0$ on $\partial \Omega$, it results that that $\bar{p}_{1}=0=\bar{p}_{2}$ and $\bar{p}_{3} \in H_{0}^{1}(\omega)$. Then, (5.28) holds true with $\bar{q}=\bar{p}_{3}$.

To prove (5.29), for for every $n \in \mathbb{N}$ let

$$
m_{n, 3}: x^{\prime} \in \omega \rightarrow \int_{-\frac{1}{2}}^{\frac{1}{2}} p_{n, 3}\left(x^{\prime}, x_{3}\right) \mathrm{d} x_{3} .
$$

By using the Poincaré-Wirtinger inequality and noticing that $p_{n} \wedge \nu=0$ on $\partial \Omega$ entails $\left(p_{n, 1}\left(x_{1}, x_{2}, \cdot\right)\right.$, 
$\left.p_{n, 2}\left(x_{1}, x_{2}, \cdot\right)\right) \in\left(H_{0}^{1}(]-\frac{1}{2}, \frac{1}{2}[)\right)^{2}$, there exists a positive constant $c$ such that, for $x^{\prime}$ a.e. in $\omega$,

$$
\begin{gathered}
\left\|\frac{1}{h_{n}} p_{n, i}\left(x^{\prime}, \cdot\right)\right\|_{H_{0}^{1}(]-\frac{1}{2}, \frac{1}{2}[)} \leq \frac{c}{h_{n}}\left\|\frac{\partial p_{n, i}\left(x^{\prime}, \cdot\right)}{\partial x_{3}}\right\|_{L^{2}(]-\frac{1}{2}, \frac{1}{2}[)}, \quad \forall n \in \mathbb{N}, \quad i=1,2, \\
\left\|\frac{1}{h_{n}}\left(p_{n, 3}\left(x^{\prime}, \cdot\right)-m_{n, 3}\left(x^{\prime}\right)\right)\right\|_{H_{m}^{1}(]-\frac{1}{2}, \frac{1}{2}[)} \leq \frac{c}{h_{n}}\left\|\frac{\partial p_{n, 3}\left(x^{\prime}, \cdot\right)}{\partial x_{3}}\right\|_{L^{2}(]-\frac{1}{2}, \frac{1}{2}[)}, \quad \forall n \in \mathbb{N} .
\end{gathered}
$$

Thus, integrating these inequalities over $x^{\prime} \in \omega$, estimate (5.30) gives (5.29).

The second step is devoted to identify $\bar{q}$ and $z$. Since $(0,0, q) \in S$ for every $q \in H_{0}^{1}(\omega)$, it results that

$$
E_{n}\left(p_{n}\right) \leq E_{n}((0,0, q)) \quad \forall q \in H_{0}^{1}(\omega), \quad \forall n \in \mathbb{N} .
$$

Then, passing to the limit in (5.31), by virtue of (3.6), Proposition 4.1, (5.28), (5.29) and a l.s.c. argument one obtains that

$$
\begin{array}{r}
\int_{\Omega}\left[\beta\left(\left|\frac{\partial \bar{q}}{\partial x_{2}}-\frac{\partial z_{2}}{\partial x_{3}}\right|^{2}+\left|\frac{\partial z_{1}}{\partial x_{3}}-\frac{\partial \bar{q}}{\partial x_{1}}\right|^{2}\right)+\right. \\
\left.\left|\frac{\partial z_{3}}{\partial x_{3}}\right|^{2}\right] \mathrm{d} x+\int_{\omega}\left[\alpha\left(|\bar{q}|^{2}-1\right)^{2}+|\bar{q}|^{2}\right] \mathrm{d} x^{\prime}-\int_{\omega}\left(\int_{-\frac{1}{2}}^{\frac{1}{2}} f_{3} \mathrm{~d} x_{3} \bar{q}\right) \mathrm{d} x^{\prime} \\
\leq \liminf _{n} E_{n}\left(p_{n}\right) \leq \underset{n}{\limsup } E_{n}\left(p_{n}\right) \leq M_{\infty}(q), \forall q \in H_{0}^{1}(\omega)
\end{array}
$$

On the other hand, since $\bar{q}$ is independent of $x_{3}$ and $\left(z_{1}, z_{2}\right) \in\left(L^{2}\left(\omega, H_{0}^{1}(]-\frac{1}{2}, \frac{1}{2}[)\right)\right)^{2}$, it results that

$$
\int_{\Omega}\left(\left|\frac{\partial \bar{q}}{\partial x_{2}}-\frac{\partial z_{2}}{\partial x_{3}}\right|^{2}+\left|\frac{\partial z_{1}}{\partial x_{3}}-\frac{\partial \bar{q}}{\partial x_{1}}\right|^{2}\right) \mathrm{d} x=\int_{\omega}|D \bar{q}|^{2} \mathrm{~d} x^{\prime}+\int_{\Omega}\left(\left|\frac{\partial z_{1}}{\partial x_{3}}\right|^{2}+\left|\frac{\partial z_{2}}{\partial x_{3}}\right|^{2}\right) \mathrm{d} x
$$

Hence, inserting (5.33) in (5.32), one has that

$$
c \int_{\Omega}\left|\frac{\partial z}{\partial x_{3}}\right|^{2} \mathrm{~d} x+M_{\infty}(\bar{q}) \leq \liminf _{n} E_{n}\left(p_{n}\right) \leq \limsup _{n} E_{n}\left(p_{n}\right) \leq M_{\infty}(q), \forall q \in H_{0}^{1}(\omega),
$$

where $c=\min \{1, \beta\}$, which entails that

$$
\frac{\partial z}{\partial x_{3}}=0, \text { a.e. in } \Omega
$$

(in particular, $z=0$ a.e. in $\Omega$ since $z \in\left(L^{2}\left(\omega, H_{0}^{1}(]-\frac{1}{2}, \frac{1}{2}[)\right)\right)^{2} \times L^{2}\left(\omega, H_{m}^{1}(]-\frac{1}{2}, \frac{1}{2}[)\right)$ ). Consequently, inserting (5.35) in (5.34), one obtains that $\bar{q}$ solves problem (5.26) and convergence (5.27) holds true. One can conclude arguing as in the end of the Proof of Theorem 5.1.

Remark 5.4. Let $\bar{S}_{\infty}=\{(0,0)\} \times\left\{q \in H^{1}(\Omega): q\right.$ independent of $x_{3}, q=0$ on $\left.\partial \omega \times\right]-\frac{1}{2}, \frac{1}{2}[\}$ and $\bar{M}_{\infty}(p)=$ $\left\{\begin{array}{l}M_{\infty}(p) \text { in } \bar{S}_{\infty}, \\ +\infty \text { in } S \backslash \bar{S}_{\infty} .\end{array}\right.$ Then, $\Gamma$-convergence of $E_{\left.n\right|_{S}}$ to $\bar{M}_{\infty}$ in the strong topology of $\left(L^{2}(\Omega)\right)^{3}$ follows easily from previous proofs.

Acknowledgements. The authors wish to thank "CMA" of "École Polytechnique" (Palaiseau - France), where the first author was invited as visiting professor in May 2010 and where this research started. This paper is part of project "MICINN MTM 2009-12628" and of the Italian projet PRIN 2008: "Modelli avanzati per strutture sottili composite". 


\section{REFERENCES}

[1] F. Alouges and S. Labbé, Convergence of a ferromagnetic film model. C. R. Math. Acad. Sci. Paris 344 (2007) $77-82$.

[2] H. Ammari, L. Halpern and K. Hamdache, Asymptotic behavior of thin ferromagnetic films. Asymptot. Anal. 24 (2000) $277-294$.

[3] G. Carbou, Thin Layers in Micromagnetism. Math. Model. Methods Appl. Sci. 11 (2001) 1529-1546.

[4] P. Chandra and P.B. Littlewood, A Landau primer for ferroelectrics, The Physics of ferroelectrics: A modern perspective, edited by K. Rabe, C.H. Ahn and J.-M. Triscone. Topics Appl. Phys. 105 (2007) 69-116.

[5] P.G. Ciarlet and P. Destuynder, A Justification of the two-dimensional linear plate model. J. Méca. 18 (1979) $315-344$.

[6] M. Costabel, M. Dauge and S. Nicaise, Singularities of Maxwell interface problems. ESAIM: M2AN 33 (1999) 627-649.

[7] E. De Giorgi and T. Franzoni, Su un tipo di convergenza variazionale. Atti Accad. Naz. Lincei Rend. Cl. Sci. Fis. Mat. Natur. 58 (1975) 842-850.

[8] A. Desimone, R.V. Kohn, S. Muller and F. Otto, A reduced theory for thin-film micromagnetics. Commun. Pure Appl. Math. 55 (2002) 1408-1460.

[9] A. Gaudiello and R. Hadiji, Junction of ferromagnetic thin films. Calc. Var. Partial Differ. Equ. 39 (2010) $593-619$.

[10] G. Gioia and R.D. James, Micromagnetism of very thin films. Proc. of R. London A 453 (1997) $213-223$.

[11] R.V. Kohn and V.V. Slastikov, Another thin-film limit of micromagnetics. Arch. Ration. Mech. Anal. 178 (2005) $227-245$.

[12] R.C. Smith, Smart material systems. model development, in Front. Appl. Math. Vol. 32. SIAM (2005).

[13] Y. Su and C.M. Landis, Continuum thermodynamics of ferroelectric domain evolution: theory, finite element implementation, and application to domain wall pinning. J. Mech. Phys. Solids 55 (2007) 280-305.

[14] W. Zhang and K. Bhattacharya, A computational model of ferroelectric domains. Part I. Model formulation and domain switching. Acta Mater. 53 (2005) 185-198. 